\title{
Effects of Cilostazol, a Selective Cyclic AMP Phosphodiesterase Inhibitor on Isolated Rabbit Spinal Arterioles
}

\author{
Yasuaki YASHIRO and Toshio OHHASHI \\ 1st Department of Physiology, Shinshu University School of Medicine, Matsumoto, 390-8621 Japan
}

\begin{abstract}
Cilostazol, a potent inhibitor of guanosine $3^{\prime}: 5^{\prime}$-cyclic monophosphate (cGMP)inhibited adenosine $3^{\prime}: 5^{\prime}$-cyclic monophosphate (cAMP) phosphodiesterase (PDE3), has been used clinically for the treatment of chronic peripheral arterial occlusive disease. The beneficial effect of cilostazol is attributed to both anti-platelet aggregating activity and vasodilation. However, the effect of cilostazol on resistance-sized vasculature is not well documented. Furthermore, mechanisms of vasodilation and influence on endothelium function are not fully understood. Thus, we investigated the vasodilator action of cilostazol using isolated, pressurized rabbit spinal arterioles with special reference to the functional endothelium. Cilostazol, acetylcholine (ACh), isocarbacyclin (prostacyclin analogue), and sodium nitroprusside (SNP) all produced concentration-dependent vasodilations of isolated spinal arterioles with endogenous myo-
\end{abstract}

genic tone. The order of potency of these agonists was isocarbacyclin $>$ ACh $>$ SNP $>$ cilostazol. Indomethacin $(10 \mu \mathrm{M}$, a cyclo-oxygenase inhibitor), $\mathrm{N}^{\omega}$-nitro-L-arginine methyl ester (LNAME, a nitric oxide synthase inhibitor, $30 \mu \mathrm{M}$ ), or chemical denudation of the endothelial cells did not significantly alter the cilostazol-induced arteriolar dilation. Furthermore, stimulating the release of endothelium-derived relaxing factors by administering ACh (100 nM), or treating with isocarbacyclin (1 nM) or SNP (3 nM) did not significantly modify the cilostazol-induced vasodilation. These results suggest that cilostazol produces the vasodilation of isolated, pressurized rabbit spinal arterioles independent of the functional endothelium. We infer that the vasodilator action of cilostazol in the spinal arterioles may be attributed to a yet unknown mechanism that is independent of the PDE3 inhibition. [Japanese Journal of Physiology, 52, 471-477, 2002]

Key words: spine, arteriole, cilostazol, vasodilation, endothelium.

Cilostazol (6-[4-(1-cyclohexyl-1H-tetrazol-5-yl)butoxy]-3,4-dihydro-2(1H)-quinolinone, OPC-13013) is an adenosine $3^{\prime}: 5^{\prime}$-cyclic monophosphate (cAMP) phosphodiesterase (PDE) inhibitor known especially as a potent antithrombotic drug, which exerts a selective inhibition of guanosine $3^{\prime}: 5^{\prime}$-cyclic monophosphate (cGMP)-inhibited cAMP phosphodiesterase (PDE3) in platelets [1-3]. Cilostazol has been used clinically for the treatment of chronic arterial occlusive disease [4]. Several clinical trials have shown that cilostazol significantly improves exercise performance in intermittent claudication, the most common symptom of lower-extremity peripheral artery occlusive disease [5-8]. A recent clinical trial using a randomized, placebo-controlled, double-blind method also showed that long-term administration of cilostazol was safe and effective in preventing the recurrence of cerebral infarction, especially lacunar infarction [9]. The mechanism by which cilostazol benefits the cerebral circulation is not fully understood. However, the vasorelaxant effect of cilostazol, as well as the inhibition of platelet aggregation may, in part, contribute to its beneficial action. In support of this idea, cilostazol was reported to dilate pial arteries and inhibit the formation of thrombi during focal ischemia in the cat brain in vivo [10]. In humans, long-term oral adminis-

Received on March 14, 2002; accepted on September 17, 2002

Correspondence should be addressed to: Toshio Ohhashi, 1st Department of Physiology, Shinshu University School of Medicine, 3-1-1 Asahi, Matsumoto, 390-8621 Japan. Tel: +81-263-37-2595, Fax: +81-263-36-5149, E-mail: ohhashi@sch.md.shinshu-u.ac.jp 
tration of cilostazol was demonstrated to increase cerebral blood flow in patients with chronic cerebral infarction [11]. Since lacunar infarction is defined as a small deep infarction that involves occlusion of small penetrating arterioles [12], it is of interest whether or not cilostazol elicits cerebral vasodilation at the arteriolar level. Plus, in spite of the numerous clinical and experimental findings on cilostazol, little information exists regarding the mechanisms by which cilostazol exerts vasodilation in arterioles.

Cilostazol elicits an anti-thrombotic effect via the inhibition of PDE3 and subsequent increase of cAMP in platelets. By the same logic, the vasodilator action of cilostazol is assumed to be due to PDE3 inhibition and corresponding cAMP increase in smooth muscles since PDE3 is strongly expressed in smooth muscle cells $[13,14]$. Endothelium releases endogenous vasodilators such as nitric oxide (NO) and vasodilator prostaglandins in response to various chemical and mechanical stimuli. NO is well known to activate guanylate cyclase in vascular smooth muscles and increase cGMP in the cells, which may theoretically attenuate the PDE3 activity. Vasodilator prostaglandins, on the other hand, may potentiate the vasodilator action of cilostazol by increasing cAMP in the smooth muscles via activating adenylate cyclase [15-17]. Thus, although cilostazol and other PDE3 inhibitors are shown to exert vasodilation even in the absence of intact endothelium [10,13,18, 19], it is of particular interest whether or not endothelium or endotheliumderived factors can modulate the vasodilator action of cilostazol.

Our laboratory has been studying spinal circulation in dogs [20,21] and rabbits [22], which could have significant physiological and clinical relevance as a regulator of the central nervous system. We found that isolated, pressurized arterioles from the rabbit spine are a highly suitable preparation for an in vitro analysis of arterioles because: (1) It is relatively easy to dissect as it possesses little connective tissues surrounding the vessel; (2) the distance between branches is substantially long $(1-2 \mathrm{~mm})$, which is perfect for cannulation at both sides without leakage; and (3) arterioles develop spontaneous myogenic tone $(\sim 60 \%$ of the passive diameter), which allows studies of vadodilator agents without any agonist-induced preconstriction. The data obtained with spinal arterioles may be extrapolated to cerebral microcirculation as the same arteriole feeding the central nervous system. We have therefore attempted to examine the effects of cilostazol on pressurized arterioles $(\sim 110 \mu \mathrm{m}$ passive diameter) isolated from rabbit spine with special reference to $\mathrm{NO}$ and vasodilator prostaglandins.

\section{MATERIALS AND METHODS}

Preparation and dissection. The Shinshu University School of Medicine Animal Ethics Committee approved the procedures and protocols used in this study. Nineteen Japanese white rabbits (1-3month-old, $1.5-3.0 \mathrm{~kg}$ ) were anesthetized with pentobarbital sodium $(40 \mathrm{mg} / \mathrm{kg}$, I.V.) and exanguinated. Detailed methods for the dissection and cannulation of the spinal arterioles have been described previously [22]. Briefly, the spinal cord (lumbar portion) was rapidly removed and placed in a cooled $\left(4^{\circ} \mathrm{C}\right)$ dissection chamber filled with 3-(N-morpholino) propanesulfonic acid (MOPS)-buffered physiological salt solution containing $1 \%$ dialyzed bovine serum albumin (BSA). A spinal arteriole $(\sim 110 \mu \mathrm{m}$ in passive diameter and $\sim 3 \mathrm{~mm}$ long) was carefully dissected and transferred to a temperature-controlled cannulation chamber mounted on the stage of an inverted microscope (model IMT-2, Olympus, Tokyo, Japan). The arteriole was cannulated at both ends with a system of concentric glass pipettes (a perfusion pipette within a holding pipette) and connected to water manometers used to adjust intraluminal pressure via independent reservoirs (Fig. 1). After the arteriole was set to its in situ length, the intraluminal pressure was set at $60 \mathrm{mmHg}$ [22]. Upstream and downstream reservoirs were set at the same height so that no flow is established in the lumen. The organ chamber was gradually heated to $37.0 \pm 0.5^{\circ} \mathrm{C}$, and was suffused with MOPS solution without albumin at a constant flow rate $(9 \mathrm{ml} / \mathrm{min})$. The image of the pressurized arteriole was

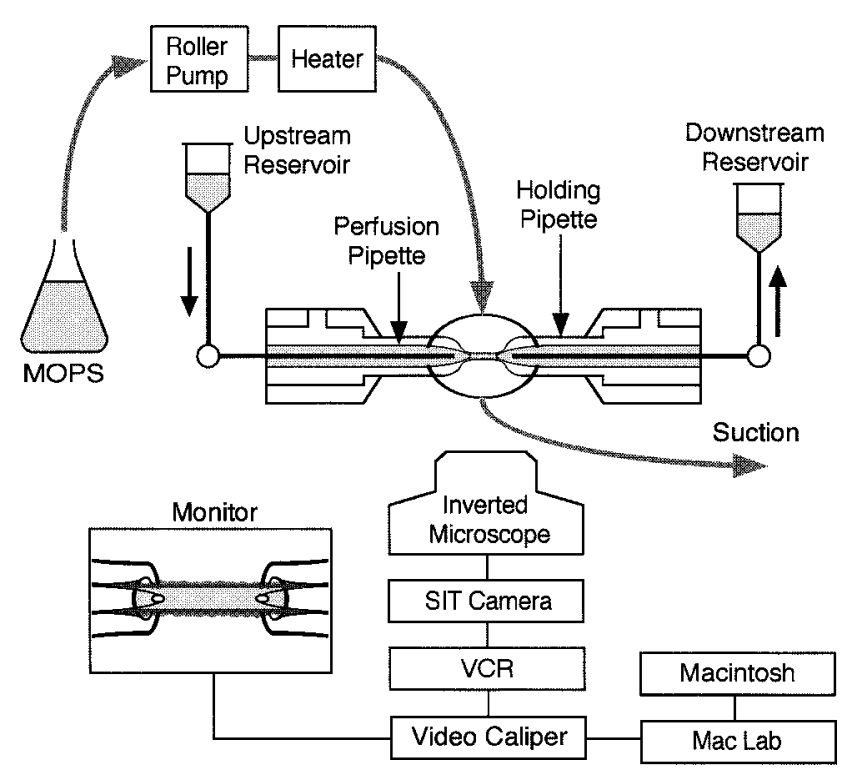

Fig. 1. Experimental layout of cannulation system, tissue chamber, and apparatus for measurement of internal diameter of the isolated arterioles. 
displayed on a television monitor, and luminal diameters were measured manually using a video caliper incorporated with MacLab Chart v3.2 (AD Instrument Inc., Castle Hill, Australia).

Experimental protocols. The arterioles were equilibrated for at least $60 \mathrm{~min}$, during which time they developed a myogenic tone. After equilibration, three sets of experiments were performed. In the first series, changes in the diameter of arterioles were measured before and during the application of cilostazol, acetylcholine (ACh), isocarbacyclin (a stable prostacyclin analogue), or sodium nitroprusside (SNP), and dose-response curves for these agents were compared. These drugs were applied cumulatively into the organ chamber from the extraluminal side by 10 -fold concentration increments $\left(10^{-9}-10^{-4} \mathrm{M}\right)$. The arterioles were allowed to stabilize for $4 \mathrm{~min}$ at each drug concentration. In the second series, responses of the arterioles to cilostazol were examined during extraluminal administration of cyclo-oxygenase inhibitor, indomethacin $(10 \mu \mathrm{M})$ or $\mathrm{NO}$ synthase inhibitor, $\mathrm{N}^{\omega}$ nitro-L-arginine methyl ester (L-NAME, $30 \mu \mathrm{M}$ ) or after chemical denudation of endothelium with Triton $\mathrm{X}-100(0.01 \%)$ [22]. The concentration of indomethacin and L-NAME was high enough to eliminate the arachidonic acid-induced vasodilation or ACh-induced NO-dependent vasodilation, respectively [22]. Successful denudation of the endothelium was confirmed by loss of vasodilation in response to $10 \mu \mathrm{M}$ ACh. Indomethacin and L-NAME were suffused for at least $30 \mathrm{~min}$ before the cumulative administration of cilostazol commenced. In the third series, dose-response curves for cilostazol were obtained in the presence of ACh, isocarbacyclin, or SMP at a concentration that induces threshold-level vasodilation ( $100 \mathrm{nM}, 1 \mathrm{nM}$, and $3 \mathrm{nM}$, respectively). The experimental procedure was the same as described in the first series of experiments. At the end of each experiment, all arterioles were relaxed completely with $10 \mu \mathrm{M}$ nifedipine to obtain maximum diameter at $60 \mathrm{mmHg}$ intraluminal pressure.

Drugs and solutions. The composition of the MOPS solution (in $\mathrm{mM}$ ) was as follows: $145 \mathrm{NaCl}$, $4.7 \mathrm{KCl}, 2.0 \mathrm{CaCl}_{2}, 1.17 \mathrm{MgSO}_{4}, 2.0$ pyruvate, 5.0 glucose, $0.02 \mathrm{EDTA}$, and 2.0 MOPS. The $\mathrm{pH}$ was adjusted to $7.4 \pm 0.02$ at $37^{\circ} \mathrm{C}$. The solution used for dissection, cannulation, and perfusion was MOPS containing 1\% BSA. Cilostazol was donated by Otsuka Pharmaceutical Co. (Tokyo, Japan). ACh chloride and isocarbacyclin were obtained from Daiichi Pharmaceutical Co. (Tokyo, Japan) and Teijin (Tokyo, Japan), respectively. Indomethacin, L-NAME hydrochloride, and Triton X-100 were obtained from Sigma Chemi- cal (St. Louis, MO, USA). Cilostazol was dissolved in $\mathrm{N}, \mathrm{N}$-dimethylformamide, and indomethacin and isocarbacyclin were dissolved in ethanol; these drugs were diluted with MOPS solution just before use. Each solvent at the final experimental concentration was confirmed to produce no significant effect on the diameter of pressurized arterioles. The other drugs were directly dissolved in the MOPS solution.

Statistics. Experimental data in the text, figures, and table are expressed as means \pm standard error of mean (SEM). The diameter changes induced by agonists were normalized to the maximum dilation induced by nifedipine $(10 \mu \mathrm{M})$ from the control diameter and are expressed as a percentage of the nifedipine-induced maximum dilation. Control diameter was defined as the diameter immediately before determination of arteriolar response to vasoactive agents. From one animal, one to three vessel segments were dissected for study. The $n$ value represents the number of vessel preparations used. The $\mathrm{p} D_{2}$ value means the negative logarithm of $\mathrm{ED}_{50}$, the concentration of agonists causing one-half maximum dilation. The vasodilation to various agonists and vasodilation before and after pharmacological interventions were analyzed with repeated measures analysis of variance (ANOVA) followed by Scheffe's test. Comparison of basal diameter before and after pharmacological treatments was performed with paired Student's $t$-test. A value of $p<0.05$ was considered significant.

\section{RESULTS}

The passive diameters of spinal arterioles at $60 \mathrm{mmHg}$ internal pressure were $110.8 \pm 2.2 \mu \mathrm{m}(n=31)$. After equilibration, arterioles developed myogenic tone and were constricted to $62.3 \pm 1.2 \%$ of their maximum (passive) diameter.

\section{Relaxant properties of spinal arterioles to va- soactive agents}

Figure 2 represents typical spinal arteriolar dilation in response to cilostazol, and Fig. 3 demonstrates dose-response curves for the four vasodilator substances. Administration of cilostazol, ACh, isocarbacyclin, and SNP all produced dose-dependent vasodilations. The difference between the two concentration-response curves was statistically significant in all of the pairs among the four agonists $(p<0.01)$. The order of potency was found to be isocarbacyclin $>$ $\mathrm{ACh}>\mathrm{SNP}>$ cilostazol. The $E_{\max }$ and $\mathrm{p} D_{2}$ values for these agents are summarized in Table 1. 
Effects of indomethacin, L-NAME, or removal of the endothelium on cilostazol-induced arteriolar dilation

The effects of $10 \mu \mathrm{M}$ indomethacin or $30 \mu \mathrm{M} \mathrm{L}$ -

\section{Control}

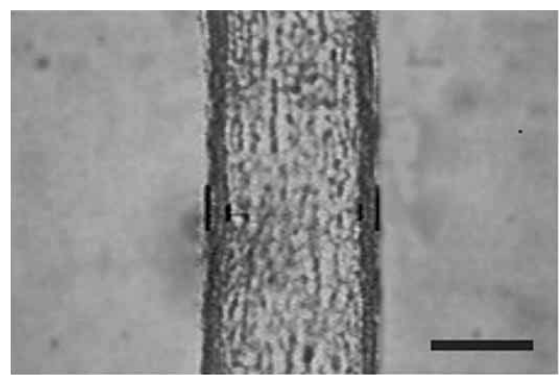

\section{$10 \mu \mathrm{M}$ Cilostazol}

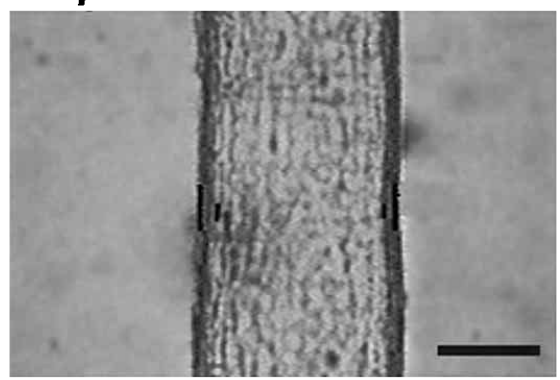

$100 \mu \mathrm{M}$ Cilostazol

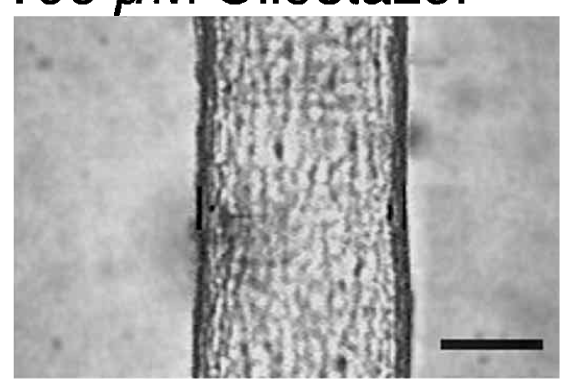

Fig. 2. Typical responses of a pressurized rabbit spinal arteriole to cumulative administrations of cilostazol. In this preparation, initial internal diameter before the pharmacological intervention was $72 \mu \mathrm{m}$ (control). During administration of 10 and $100 \mu \mathrm{M}$ cilostazol, vessel diameter increased to 90 and $96 \mu \mathrm{m}$, respectively. Bar: $50 \mu \mathrm{m}$.
NAME on cilostazol-induced vasodilation in the pressurized spinal arterioles are summarized in Fig. 4A, B. Incubation with indomethacin or L-NAME did not significantly alter the baseline diameter $(65.6 \pm 1.7 \mu \mathrm{m}$ before vs. $61.8 \pm 3.2 \mu \mathrm{m}$ after indomethacin, and $66.8 \pm 2.1 \mu \mathrm{m}$ before vs. $65.4 \pm 2.8 \mu \mathrm{m}$ after L-NAME; $n=5$, respectively), and neither treatment significantly influenced the dose-response curve for cilostazol. Chemical denudation of the endothelial layers with $0.01 \%$ Triton X-100 caused a significant decrease in the baseline diameter, $13 \%(67.0 \pm 3.1 \mu \mathrm{m}$ before vs. $58.3 \pm 3.5 \mu \mathrm{m}$ after denudation, $p<0.01 ; n=4)$. However, the removal of endothelium had a negligible effect on the cilostazol-induced arteriolar vasodilation as shown in Fig. 4C.

\section{Effects of ACh, isocarbacyclin, or SNP}

The effects of threshold levels of ACh, isocarbacyclin or SNP are summarized in Fig. 5A-C. Treatment with $100 \mathrm{nM}$ ACh slightly, but significantly, increased the baseline diameter $(66.6 \pm 5.2 \mu \mathrm{m}$ vs. $69.2 \pm 5.7$ $\mu \mathrm{m}, p<0.05 ; n=5)$. The presence of $\mathrm{ACh}$, however, did not affect the concentration-response curves produced by cilostazol (Fig. 5A). Isocarbacyclin (1 nM) also increased the baseline diameter $(63.1 \pm 3.4 \mu \mathrm{m}$ vs.

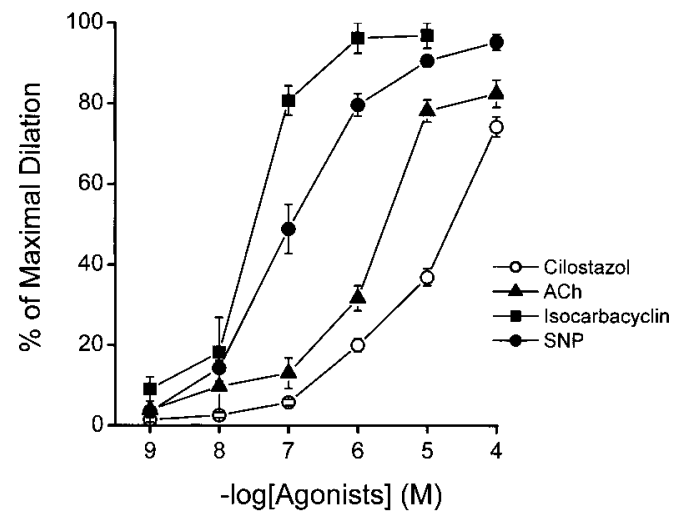

Fig. 3. The dilator responses to cilostazol $(n=16)$, acetylcholine (ACh, $n=5)$, isocarbacyclin $(n=5)$, and sodium nitroprusside (SNP, $n=5$ ) in pressurized spinal arterioles. Values are means \pm SE.

Table 1. The $E_{\max }$ and $\mathrm{p} D_{2}$ values for cilostazol, acetylcholine (ACh), isocarbacyclin, and sodium nitroprusside (SNP) in pressurized rabbit spinal arterioles.

\begin{tabular}{lcccc}
\hline $\begin{array}{c}\text { Agonist } \\
(n)\end{array}$ & $\begin{array}{c}\text { Resting diameter } \\
(\mu \mathrm{m})\end{array}$ & $\begin{array}{c}\text { Passive diameter } \\
(\mu \mathrm{m})\end{array}$ & $\begin{array}{c}E_{\max } \\
(\%)\end{array}$ & $\mathrm{p} D_{2}$ \\
\hline Cilostazol (16) & $65.9 \pm 2.7$ & $107.2 \pm 2.8$ & $74.3 \pm 2.5$ & $4.7 \pm 0.1$ \\
ACh (5) & $66.8 \pm 2.9$ & $113.2 \pm 6.1$ & $82.5 \pm 3.4^{\star \star}$ & $5.6 \pm 0.0^{\star \star}$ \\
Isocarbacyclin (5) & $72.4 \pm 4.0$ & $110.2 \pm 5.3$ & $96.9 \pm 3.1^{\star *}$ & $7.5 \pm 0.1^{\star \star}$ \\
SNP (5) & $79.8 \pm 10.4$ & $120.6 \pm 6.5$ & $95.3 \pm 2.0^{\star \star}$ & $7.0 \pm 0.1^{\star \star}$
\end{tabular}

Values are mean \pm SE. $n$, number of isolated arterioles. $E_{\max }$ is expressed as $\%$ of nifedipine-induced maximum dilation. ${ }^{* *} p<0.01$ vs. the value for cilostazol. 

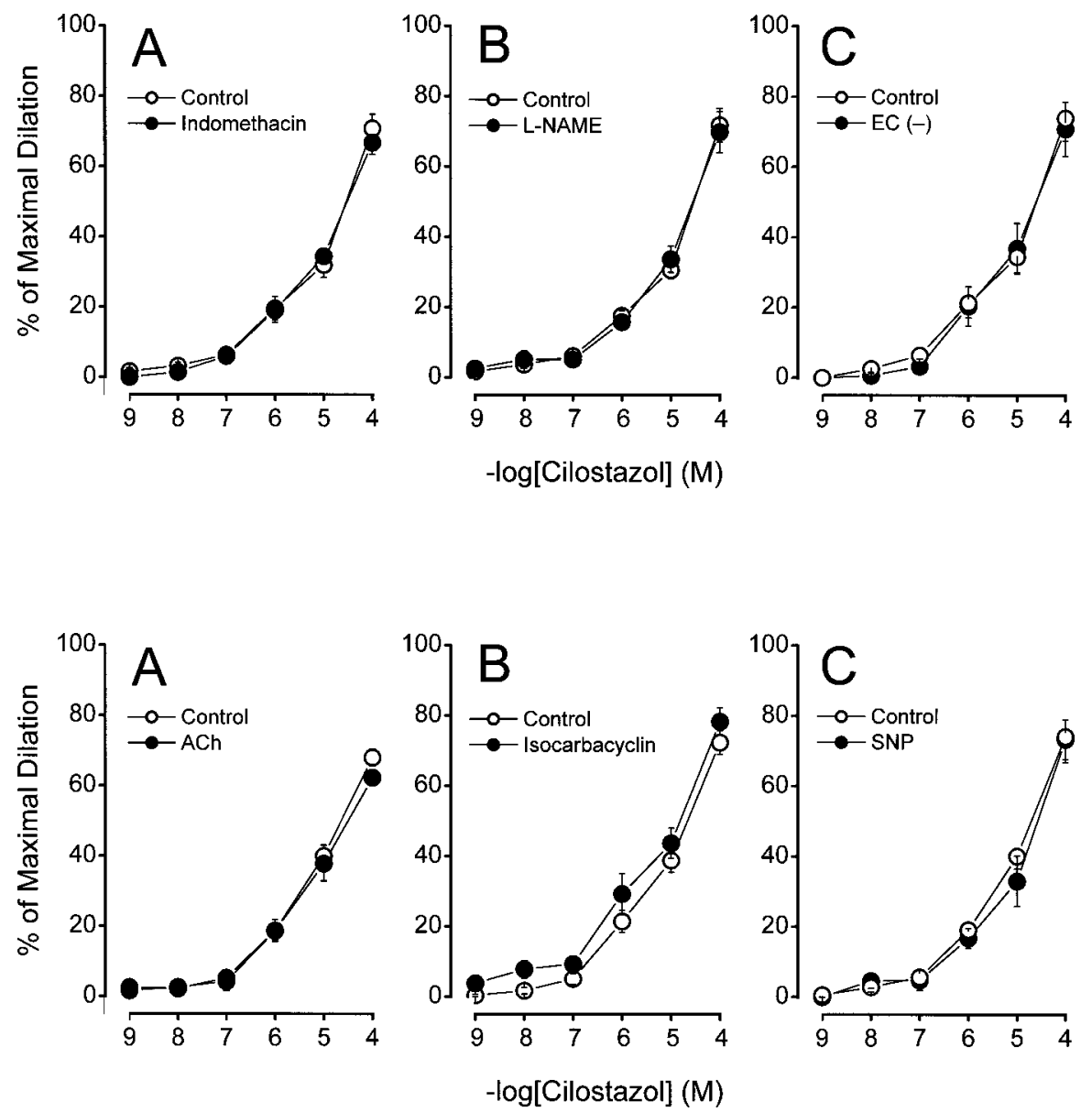

Fig. 4. Concentration-response curves for cilostazol in pressurized spinal arterioles before $(\bigcirc)$ and during (O) pharmacological blockade of cyclooxygenase or nitric oxide synthase, or before $(O)$ and after $(\Theta)$ chemical denudation of endothelium. A: Before and during treatment with indomethacin (10 $\mu \mathrm{M}, n=5)$. B: Before and during incubation of $\mathrm{N}^{\omega}$-nitro-L-arginine methyl ester (L-NAME; $30 \mu \mathrm{M}, n=5$ ). C: Before and after chemical denudation of endothelium with Triton $\mathrm{X}-100 \quad(0.01 \%, n=4)$. Values are means \pm SE.

Fig. 5. Concentration-response curves for cilostazol in pressurized spinal arterioles in the absence $(\bigcirc)$ or presence $(\Theta)$ of ACh $(100 \mathrm{nM}, \quad n=5), \quad$ isocarbacyclin ( $1 \mathrm{nM}, n=7)$, or SNP (3 nM, $n=4)$; each concentration of which produces a threshold level of vasodilation. A: Before and during presence of ACh. B: In the absence and presence of isocarbacyclin. C: Before and during administration of SNP. Values are means \pm SE.
$65.3 \pm 3.6 \mu \mathrm{m}, p<0.01 ; n=7$ ). Isocarbacyclin slightly shifted the concentration-response curve for cilostazol to the left as shown in Fig. 5B, but statistical analysis revealed no significant difference between the two curves. SNP also produced a slight increase in baseline diameter, though it was not statistically significant (66.6 $\pm 5.2 \mu \mathrm{m}$ vs. $69.2 \pm 5.7 \mu \mathrm{m} ; n=4)$. SNP had no discernible influence on cilostazol-induced vasodilation in the spinal arterioles (Fig. 5C).

\section{DISCUSSION}

Our major findings in the present study are summarized as follows: (1) Cilostazol exerts mild vasodilation of pressurized arterioles isolated from rabbit spine in a concentration-dependent manner; (2) the drug produces vasodilation even during inhibition of cyclooxygenase or NO synthase, or after the chemical denudation of endothelial cells; and (3) simulating the release of endogenous vasodilators with $\mathrm{ACh}$, or the administration of a prostacyclin analogue or a $\mathrm{NO}$ donor does not modify the efficacy of cilostazol. These results suggest that cilostazol produces vasodilation independent of the presence or activation of the endothelium in rabbit spinal arterioles. To the best of our knowledge, this is the first demonstration of the effect of cilostazol in pressurized arterioles in vitro.

Vasodilator action of cilostazol on resistance vessels. Cilostazol produced a dose-dependent dilation of pressurized spinal arterioles, although the dilator effect was mildest among the four vasodilators studied. Previously, cilostazol was shown to increase blood flow in the finger of diabetic patients with peripheral vascular disease, as evidenced by an increase in digital skin temperature [23]. In anesthetized dogs, cilostazol decreases blood pressure by reducing resistance in the peripheral blood vessels [24]. These findings support our conclusion that cilostazol can act directly on the resistance-sized vessels to alter peripheral blood flow. A recent clinical trial showed that cilostazol significantly reduces the recurrence of lacunar infarction, which involves occlusion of resistance-sized vessels in the brain [9]. Another study also indicated that long-term oral administration of cilostazol improved cerebral blood flow in patients with cerebral infarction [11]. Our findings in the present study support the idea that the vasodilator action of cilostazol at the arteriolar level may, at least in part, contribute to the beneficial effect of cilostazol preventing secondary cerebral infarction. 
Mechanisms of arteriolar dilation with
cilostazol: negligible modulation with en-
dothelium, endogenous nitric oxide, and
prostaglandins. The present study indicates that prostaglandins. The present study indicates that
cilostazol exerts the vasodilation of pressurized spinal arterioles independent of endogenous nitric oxide and vasodilator prostaglandins since indomethacin, LNAME, and even chemical denudation of endothelial cells did not significantly modify the response. The concentration of inhibitors used in the present study is high enough to block cyclo-oxygenase and nitric oxide synthase activity since the co-administration of $10 \mu \mathrm{M}$ indomethacin and $30 \mu \mathrm{M}$ L-NANE completely eliminated ACh-induced dilation of the spinal arterioles [22]. Nitric oxide activates soluble guanylate cyclase in vascular smooth muscles and increases cGMP in the cells. The increased level of cGMP could inactivate PDE3, thereby increasing the cAMP concentration in the cells and causing further vasodilation. Endogenous nitric oxide may thus compete with cilostazol against the PDE3 inhibition and reduce the efficacy of cilostazol. Vasodilator prostaglandins, on the other hand, increase the cAMP in smooth muscles via activating of adenylate cyclase [15-17]. Thus, endogenous prostanoids may potentially increase the dilator effect of cilostazol. Igawa et al. [25] demonstrated a synergistic action of cilostazol with endothelium on anti-platelet aggregating activity, and aspirin reversed the potentiated inhibitory effect of cilostazol. Such potentiation between cilostazol and endothelium on arteriolar dilation, however, was not apparent in the present study. Our results are in agreement with the report by Shigemori et al. [18] that cilostazol exerts vasodilation in the rat pulmonary arterial ring preconstricted with phenylephrine regardless of the endothelium function. On the other hand, Nakamura et al. [26] recently suggested that the vasodilator action of cilostazol is partially endothelium dependent, and that NO production is increased with cilostazol in the rat thoracic aorta.

Our results may suggest that endogenous vasodilators from intact endothelium released at rest are not high enough to modulate the action of cilostazol. To address this issue, we attempted to stimulate endothelium with a low dose of ACh to increase the release of endogenous endothelium-derived relaxing factors. We also tried to increase the basal level of cAMP or cGMP in the smooth muscles via the administration of a prostacyclin analogue or a NO donor. None of these treatments, however, was effective in modulating cilostazol-induced arteriolar dilation. These results may suggest that the vasodilator effect of cilostazol cannot be solely explained by PDE3 inhibition. The idea is, in part, supported by the report of Cone et al. [27], in which they compared the effects of cilostazol and milrinone, a conventional PDE3 inhibitor, on changes in cAMP levels and functions in platelets, ventricular myocytes, and coronary smooth muscle cells of rabbits. They showed that, in coronary smooth muscle cells, milrinone elevates cAMP and increases coronary blood flow; however, cilostazol did not increase intracellular cAMP while producing a comparable increase in the coronary flow. They also showed that, in rabbit ventricular myocites, cilostazol does not increase cAMP as much as milrinone does, and that the cardiotropic effect of these agents is significantly less with cilostazol. Similarly, Tanaka et al. [28] did not see an increase in intracellular cAMP after a 12min exposure of rabbit aorta smooth muscle cells to $10 \mu \mathrm{M}$ cilostazol. Lindgren et al. [29] compared OPC3911 (a PDE3 inhibitor structurally similar to cilostazol) with milrinone in rat aorta, and found the increase was significant only for milrinone. Nevertheless, OPC-3911 and milrinone had similar relaxant properties in human and rat coronary and renal arteries [30]. The data may not mean less potency of cilostazol on PDE3 inhibition, because cilostazol and milrinone concentration-dependently increased intracellular cAMP in platelets and inhibited human platelet aggregation with similar potency [27]. These findings, including ours, may support the conclusion that the anti-PDE3 effect of cilostazol is, to some extent, cell specific and that the detailed mechanisms of cilostazol action on the vascular smooth muscle remain to be elucidated.

The authors thank Mr. Nobuyuki Ono, Nagano Technical College, for his technical support. This work was supported in part by the Research Grant for Cardiovascular Diseases (9 koubo 3) from the Ministry of Health and Welfare of Japan and by a Grant-in-Aid for Scientific Research (No. 13770023) from the Ministry of Education, Culture, Sports, Science and Technology of Japan.

\section{REFERENCES}

1. Kawamura K, Watanabe K, and Kimura Y: Effect of cilostazol, a new antithrombotic drug, on cerebral circulation. Arzneimittelforschung 35: 1149-1154, 1985

2. Kimura Y, Tani T, Kanbe T, and Watanabe K: Effect of cilostazol on platelet aggregation and experimental thrombosis. Arzneimittelforschung 35: 1144-1149, 1985

3. Kohda N, Tani T, Nakayama S, Adachi T, Marukawa K, Ito $R$, Ishida $K$, Matsumoto $Y$, and Kimura $Y$ : Effect of cilostazol, a phosphodiesterase III inhibitor, on experimental thrombosis in the porcine carotid artery. Thromb Res 96: 261-268, 1999

4. Yasuda K, Sakuma M, and Tanabe T: Hemodynamic ef- 
fect of cilostazol on increasing peripheral blood flow in arteriosclerosis obliterans. Arzneimittelforschung 35: 1198-1200, 1985

5. Strandness DE Jr, Dalman RL, Panian S, Rendell MS, Comp PC, Zhang P, and Forbes WP: Effect of cilostazol in patients with intermittent claudication: a randomized, double-blind, placebo-controlled study. Vasc Endovasc Surg 36: 83-91, 2002

6. Beebe HG, Dawson DL, Cutler BS, Herd JA, Strandness DE Jr, Bortey EB, and Forbes WP: A new pharmacological treatment for intermittent claudication: results of a randomized, multicenter trial. Arch Intern Med 159: 2041-2050, 1999

7. Dawson DL, Cutler BS, Meissner MH, and Strandness DE Jr: Cilostazol has beneficial effects in treatment of intermittent claudication: results from a multicenter, randomized, prospective, double-blind trial. Circulation 98: 678-686, 1998

8. Money SR, Herd JA, Isaacsohn JL, Davidson M, Cutler B, Heckman J, and Forbes WP: Effect of cilostazol on walking distances in patients with intermittent claudication caused by peripheral vascular disease. J Vasc Surg 27: 267-274, 1998

9. Gotoh F, Tohgi H, Hirai S, Terashi A, Fukuuchi Y, Otomo E, Shinohara Y, Itoh E, Matsuda T, Sawada T, Yamaguchi T, Nishimaru K, and Ohashi Y: Cilostazol stroke prevention study: a placebo-controlled double-blind trial for secondary prevention of cerebral infarction. $J$ Stroke Cerebravasc Dis 9: 147-157, 2002

10. Tanaka K, Gotoh F, Fukuuchi Y, Amano T, Uematsu D, Kawamura J, Yamawaki T, Itoh N, Obara K, and Muramatsu K: Effects of a selective inhibitor of cyclic AMP phosphodiesterase on the pial microcirculation in feline cerebral ischemia. Stroke 20: 668-673, 1989

11. Kobayashi $S$, Yamaguchi $S$, Katsube $T$, Kitani $M$, Okada $\mathrm{K}$, and Tsunematsu $\mathrm{T}$ : Long-term effect of cilostazol on cerebral blood flow in chronic cerebral infarction. Arzneimittelforschung 35: 1193-1197, 1985

12. Bamford J, Sandercock P, Dennis M, Burn J, and Warlow C: Classification and natural history of clinically identifiable subtypes of cerebral infarction. Lancet 337 : 1521-1526, 1991

13. Komas N, Lugnier C, and Stoclet JC: Endothelium-dependent and independent relaxation of the rat aorta by cyclic nucleotide phosphodiesterase inhibitors. $\mathrm{Br} \mathrm{J}$ Pharmacol 104: 495-503, 1991

14. Komas N, Lugnier C, Andriantsitohaina R, and Stoclet JC: Characterisation of cyclic nucleotide phosphodiesterases from rat mesenteric artery. Eur J Pharmacol 208: 85-87, 1991

15. Schubert R, Serebryakov VN, Mewes $H$, and Hopp $H H$ : lloprost dilates rat small arteries: role of $\mathrm{K}_{\mathrm{ATP}}$ and $\mathrm{K}_{\mathrm{Ca}^{-}}$ channel activation by cAMP-dependent protein kinase. Am J Physiol Heart Circ Physiol 272: H1147-H1156, 1997

16. Parfenova H, Hsu P, and Leffler CW: Dilator prostanoidinduced cyclic AMP formation and release by cerebral microvascular smooth muscle cells: inhibition by indomethacin. J Pharmacol Exp Ther 272: 44-52, 1995

17. Vegesna RV and Diamond J: Elevation of cyclic AMP by prostacyclin is accompanied by relaxation of bovine coronary arteries and contraction of rabbit aortic rings.
Eur J Pharmacol 128: 25-31, 1986

18. Shigemori K, Ishizaki T, Takahashi H, Sasaki $F$, Ameshima S, Ohnishi T, Sakai T, Nakai T, and Miyabo $S$ : Effects of cilostazol, a cyclic AMP phosphodiesterase inhibitor, on pulmonary vascular tone. Jpn J Thorac Dis 31: 936-941, 1993 (in Japanese)

19. Shiraishi $Y$, Kanmura $Y$, and Itoh T: Effect of cilostazol, a phosphodiesterase type III inhibitor, on histamine-induced increase in $\left[\mathrm{Ca}^{2+}\right]_{i}$ and force in middle cerebral artery of the rabbit. $\mathrm{Br} J$ Pharmacol 123: 869-878, 1998

20. Shirai K, Kawai $Y$, and Ohhashi T: Contractile and relaxant responses of the canine isolated spinal artery to vasoactive substances. Br J Pharmacol 101: 200-204, 1990

21. Wang JX, Ikomi F, and Ohhashi T: 5-Hydroxytryptamine-induced endothelium-dependent and -independent relaxations in isolated dog anterior spinal small arteries. Can J Physiol Pharmacol 75: 357-362, 1997

22. Yashiro $Y$ and Ohhashi T: Flow- and agonist-mediated nitric oxide- and prostaglandin-dependent dilation in spinal arteries. Am J Physiol Heart Circ Physiol 273: H2217-H2223, 1997

23. Uchikawa T, Murakami T, and Furukawa H: Effects of the anti-platelet agent cilostazol on peripheral vascular disease in patients with diabetes mellitus. Arzneimittelforschung 42: 322-324, 1992

24. Shintani S, Watanabe K, Kawamura K, Mori T, Tani T, Toba Y, Sasabe H, Nakagiri N, Hongoh O, and Fujita S: General pharmacological properties of cilostazol, a new antithrombotic drug. Part II: Effect on the peripheral organs. Arzneimittelforschung 35: 1163-1172, 1985

25. Igawa T, Tani T, Chijiwa T, Shiragiku T, Shimidzu S, Kawamura K, Kato S, Unemi F, and Kimura Y: Potentiation of anti-platelet aggregating activity of cilostazol with vascular endothelial cells. Thromb Res 57: 617-623, 1990

26. Nakamura $T$, Houchi $H$, Minami A, Sakamoto $S$, Tsuchiya K, Niwa Y, Minakuchi K, and Nakaya Y: Endothelium-dependent relaxation by cilostazol, a phosphodiesteras III inhibitor, on rat thoracic aorta. Life Sci 69: 1709-1715, 2001

27. Cone J, Wang S, Tandon N, Fong M, Sun B, Sakurai K, Yoshitake M, Kambayashi J, and Liu Y: Comparison of the effects of cilostazol and milrinone on intracellular cAMP levels and cellular function in platelets and cardiac cells. J Cardiovasc Pharmacol 34: 497-504, 1999

28. Tanaka T, Ishikawa T, Hagiwara M, Onoda $K$, Itoh $H$, and Hidaka $\mathrm{H}$ : Effects of cilostazol, a selective cAMP phosphodiesterase inhibitor on the contraction of vascular smooth muscle. Pharmacology 36: 313-320, 1988

29. Lindgren $\mathrm{SH}$, Andersson TL, Vinge $\mathrm{E}$, and Andersson $\mathrm{KE}$ : Effects of isozyme-selective phosphodiesterase inhibitors on rat aorta and human platelets: smooth muscle tone, platelet aggregation and cAMP levels. Acta Physiol Scand 140: 209-219, 1990

30. Lindgren $S$ and Andersson KE: Effects of selective phosphodiesterase inhibitors on isolated coronary, lung and renal arteries from man and rat. Acta Physiol Scand 142: 77-82, 1991 